ORIGINAL ARTICLE

\title{
Do consultants differ? Inferences drawn from hospital in-patient enquiry (HIPE) discharge coding at an Irish teaching hospital
}

\author{
E D Moloney, D Smith, K Bennett, D O'Riordan, B Silke
}

Postgrad Med J 2005;81:327-332. doi: 10.1136/pgmi.2004.026245

See end of article for authors' affiliations

.....................

Correspondence to: Dr B Silke, Department of Pharmacology and Therapeutics, Trinity Centre for Health Sciences, St James' Hospital, James' Street, Dublin 8, Ireland; silkeb@tcd.ie

Submitted 29 June 2004 Accepted 17 August 2004
Objective: To find out if there was a difference between hospital consultants, all trained in acute general medicine, in length of stay (LOS), re-admission rates, resource utilisation, and diagnostic coding, among patients admitted as emergencies to St James' Hospital (SJH) Dublin.

Methods: A retrospective analysis was performed of data on discharges from hospital, recorded in the hospital in-patient enquiry (HIPE) system, relating to 9204 episodes among 6968 emergency medical patients admitted to SJH between 1 January 2002 and 31 October 2003. For comparative analysis, four physician groups were defined consisting of gastroenterology $(G I, n=4)$, respiratory $(n=3)$, general internal medicine (GIM, $n=2)$, or specialty $(n=5)$.

Results: GIM consultants had the shortest LOS (median 5 days); GIM and respiratory consultants were less likely to have long stay patients ( $>30$ days, $p<0.0001$ ). Patients re-admitted under the same consultant had a longer LOS than those re-admitted under a different consultant $(p<0.0001)$. Endoscopy and $\mathrm{Gl}$ radiology investigations were used most by $\mathrm{Gl}$ consultants, computed tomography of the thorax by respiratory, ECHO by respiratory and specialty, and computed tomography of brain by GIM and specialty consultants. GI diagnostic codings were more frequent with $G$ consultants $(p<0.0001)$, respiratory diagnoses and malignancy with respiratory $(p<0.0001$ for both), diabetes and hypertension with specialty $(p=0.0017)$, and heart failure more with GIM consultants $(p=0.001)$.

Conclusions: This study found that the HIPE database was very powerful in predicting differences between hospital consultants in LOS, re-admission rates, resource utilisation, and disease coding. It would be of interest to examine the extent to which protocols and guidelines could reduce such variations.
W hile previous studies have reported differences in length of hospital stay (LOS) and resource utilisation between various hospital physicians, ${ }^{1-6}$ there is a lack of such data between hospital consultants in the Republic of Ireland. Given growing pressures to manage costs and maximise efficiency in acute hospital services, information on patterns of resource utilisation, including LOS, by medical subspecialty is valuable. In North America, it has been suggested that physicians are responsible for an estimated $80 \%$ to $90 \%$ of hospital resource allocation charges, and determine how more than $10 \%$ of the gross national product is spent. ${ }^{4}$ Increasing specialisation and the more frequent use of expensive technologies by specialists have been cited as important factors increasing healthcare costs, and have stimulated interest in cost effective medicine..$^{5-7}$ We have previously shown a median LOS of six days for patients admitted acutely via the emergency department over a one year period to a teaching hospital in the Republic of Ireland; the LOS was shorter with admission under a general medical service. $^{8}$

Data on discharges from acute public hospitals in Ireland are recorded in the hospital in-patient enquiry (HIPE) system. Sixty hospitals participate in the system and it is an invaluable source of hospital activity level and accreditation. The recent report of the HIPE Unit, from the Economic and Social Research Institute marks an important attempt to quantify the workload and case mix in Irish hospitals. ${ }^{9}$ The principal objective of this study is to find out whether there is a difference between hospital consultants in LOS, readmission rates, resource utilisation, and diagnostic coding among patients admitted as emergencies to a general medical department of a teaching hospital in Dublin, Ireland.

\section{PATIENTS AND METHODS}

Data relating to emergency medical patients admitted to St James' Hospital between 1 January 2002 and 31 October 2003 were recorded. St James' Hospital, although a tertiary referral centre for various specialties, operates a daily sectorised acute general medical "take-in" serving as a secondary care centre for emergency medical admissions for its local Dublin catchment area. Patients were admitted directly to a variety of wards, many of which are not affiliated with a medical specialty, under the care of a named consultant physician. Fourteen different consultant physicians were responsible for the management of these patients of which 10 were whole time health service consultants and four held split service/ academic appointments. The "on-call" roster is a 1:9 with two slots each operated by teams from respiratory medicine and gastroenterology (GI), one slot each contributed by specialty teams from diabetes/endocrinology, clinical pharmacology, and rheumatology, and one slot each contributed by two teams from general internal medicine (GIM). All consultant physicians were trained in acute general medicine as well as having a subspecialty interest. For the comparative analysis between physicians, four groups were defined consisting of respiratory $(n=3)$, GI $(n=4)$, GIM $(n=2)$, or specialty $(n=5)$; the first three groups were on-call for two slots with the last occupying three of the nine slots.

The patient database was acquired by linking the patient administration system (PAS) to the HIPE scheme. HIPE is a

Abbreviations: HIPE, hospital in-patient enquiry scheme; LOS, length of hospital stay; IQR, interquartile range; GIM, general internal medicine; $\mathrm{Gl}$, gastroenterology; CT, computed tomography; PAS, patient administration system 
national database of coded discharge summaries from acute public hospitals in Ireland. Ireland uses the International Classification of Diseases, ninth revision, clinical modification (ICD-9-CM) for both diagnosis and procedure coding since 1990, with updates every five years. Sixty hospitals participate in the system nationally; the computer based discharge abstracting system is designed to collect demographic, clinical, and administrative data on discharges and deaths from acute general hospitals. It is the only source of morbidity data available nationally for acute hospital services in Ireland. Linking the HIPE dataset with the patient administration system (PAS) permits application of routinely collected data for the purposes of research, planning, and quality control. Data collected include hospital number; patient's name; dates of admission and discharge; date of birth; sex; area of residence by county; diagnosis-principal and up to nine additional secondary diagnoses; proceduresprincipal and up to nine additional secondary procedures, consultant responsible for care. The HIPE dataset of all coded diseases at time of discharge/death, together with procedures and investigations undertaken during the in-hospital stay was examined. Codes with less than 20 occurrences were not considered for analysis. Individual codes together with the combination of all related codes were evaluated.

\section{Statistical methods}

Descriptive analyses are presented in the form medians, interquartile range (IQR), and proportions. A categorical variable was computed for each patient based on whether they had a single or multiple admissions. Where there were multiple admissions, we examined the initial and first readmission, to see if each was under the care of the same consultant. As LOS was non-normal, associations were examined between LOS and categorical measures using a non-parametric Wilcoxon or Kruskall-Wallis rank sum test. The Charlson comorbidity method was used to compute a weighted index for each patient.' A higher weighting score (based on 19 diagnostic categories) indicates more comorbid disease and has been used to adjust the results for LOS and readmissions. Categorical measures were cross tabulated and where appropriate $\chi^{2}$ tests performed. Significance at $\mathrm{p}<0.05$ is assumed throughout and a Bonferonni correction made for repeated statistical testing. All analyses were performed using the JMPin statistical package (version 5.1, SAS Institute).

\section{RESULTS}

Altogether 9204 episodes were recorded among 6968 patients admitted acutely via the emergency department in the 22 month study period, I January 2002 to 31 October 2003. The median age of admissions was 65.8 years (IQR
45.1-77.5); $10 \%$ of admissions were over 84 years. Less than half of patients $(48.7 \%)$ were male. The median length of stay (LOS) was six days (IQR 2-12). Seven hundred and twenty six episodes $(8.0 \%)$ had a LOS $>30$ days. Patients were readmitted up to 28 times; 5662 patients $(81.3 \%)$ had a single admission, 871 patients $(12.5 \%)$ were admitted twice, 230 $(3.3 \%)$ on three, $98(1.4 \%)$ on four, and $54(0.8 \%)$ on five occasions. A further 53 patients $(0.8 \%)$ were admitted more than five times. Four patients $(0.06 \%)$ were re-admitted 15 or more times.

\section{Differences between consultant groups (LOS, re- admissions, and long stay patterns) (table 1)}

The median LOS differed significantly $(p<0.0001)$ between the consultant physician groups; LOS for GIM at five days (IQR 2,10) being lower that the other three groups at six days (IQR 2,12). For the 726 long stay patients with LOS $>30$ days, the frequency distribution differed significantly $(\mathrm{p}<0.0001)$ between consultant groupings; compared with an $8.0 \%$ overall, GIM was lower and specialty higher. These results remained significant after adjusting for the number of comorbid diseases

Consultant groups differed in re-admission rates $(\mathrm{p}=0.003)$ over the 22 month period; compared with an overall re-admission frequency of 39\%, respiratory consultants were higher $(41.6 \%)$ and GI lower $(36.9 \%)$. Comparing patients on their first and subsequent re-admission, there was no difference between consultant groups $(p=0.425)$. The LOS for re-admissions was significantly shorter when readmitted under the GIM teams $(\mathrm{p}<0.0001)$; compared with the overall median duration of six days (IQR 3,13) that for GIM was only five days (IQR 2,10). These results remained significant after adjusting for the number of comorbid diseases.

Patients were more likely to be re-admitted under the care of respiratory $(30 \%)$ or GI $(31.1 \%)$ consultants compared with specialty $(18.8 \%)$ or GIM $(22.3 \%)$ on the first readmission $(p=0.0003)$. Overall $25.5 \%$ of patients readmitted had the same consultant on both the initial and subsequent re-admission. The median (IQR) LOS between the same and different consultants at the first re-admission was seven days $(4,16.5)$ and six days $(2,11)$ respectively $(p<0.0001)$ - that is, re-admission under the same consultant resulted in a longer LOS on the second re-admission.

\section{Resource utilisation between consultant groups (table 2)}

There were some important differences between consultant teams in respect of resource utilisation. The use of oesophagogastroduodenoscopy (OGD) investigations was

Table 1 LOS and re-admission rates by consultant groups

\begin{tabular}{|c|c|c|c|c|c|c|c|}
\hline Variable & $\begin{array}{l}\text { GI } \\
(n=2343)\end{array}$ & $\begin{array}{l}\text { Respiratory } \\
(n=2300)\end{array}$ & $\begin{array}{l}\text { Specialty } \\
(n=2499)\end{array}$ & $\begin{array}{l}\text { GIM } \\
(n=2063)\end{array}$ & Total & p Value & $\begin{array}{l}\text { p Value adjusted } \\
\text { for Clnd }\end{array}$ \\
\hline Median LOS (IQR)* & $6(2,13)$ & $6(2,11)$ & $6(3,14)$ & $5(2,10)$ & $6(2,12)$ & $<0.0001$ & $<0.0001$ \\
\hline $\operatorname{LOS}>30$ days & $8.2 \%$ & $6.1 \%$ & $10.1 \%$ & $7.2 \%$ & $8.0 \%$ & $<0.0001$ & $<0.0001$ \\
\hline Any re-admission (\% overall) & $36.9 \%$ & $41.6 \%$ & $37.1 \%$ & $38.5 \%$ & $38.5 \%$ & 0.003 & $0.022 \ddagger$ \\
\hline Re-admission† & $13.7 \%$ & $14.5 \%$ & $14.3 \%$ & $14.4 \%$ & $14.2 \%$ & 0.425 & $0.478 \ddagger$ \\
\hline \multicolumn{8}{|l|}{ First occassion } \\
\hline Subsequent occasion & $23.2 \%$ & $27.1 \%$ & $22.8 \%$ & $24.4 \%$ & $24.3 \%$ & & \\
\hline $\begin{array}{l}\text { LOS for re-admissions* } \\
\text { Charlson index }\end{array}$ & $7(3,13)$ & $7(3,12)$ & $7(3,15)$ & $5(2,10)$ & $6(3,13)$ & $<0.0001$ & $<0.0001$ \\
\hline Median (IQR) & $0(0,1)$ & $1(0,2)$ & $1(0,1)$ & $1(0,1)$ & $1(0,1)$ & $<0.0001$ & \\
\hline Mean (SD) & $0.82(1.13)$ & $1.02(1.12)$ & $0.86(1.04)$ & $0.88(1.1)$ & $0.89(1.1)$ & & \\
\hline \multicolumn{8}{|l|}{ Charlson index } \\
\hline$\%>0$ & 46.4 & 57.3 & 52.2 & 51.8 & 51.9 & $<0.0001$ & \\
\hline
\end{tabular}

*Non-parametric Kruskall-Wallis test used for comparison of medians between groups. †Comparison between first and any subsequent readmission only $\ddagger$ Adjustment for Charlson index based on "index" admission (first admission in this case) only. LOS, length of stay; GI, gastroenterology; GIM, general internal medicine. Clnd, adjustment made for Charlson index based on all admissions. 
Table 2 Resource utilisation (\% use among consultants)

\begin{tabular}{|c|c|c|c|c|c|c|}
\hline Variable & GI & Respiratory & Specialty & GIM & Total & p Value \\
\hline OGD & 11.1 & 3.5 & 4.4 & 3.6 & 5.7 & $<0.0001$ * \\
\hline ERCP & 7.4 & 0.1 & 0.6 & 0.1 & 2.1 & $<0.0001$ * \\
\hline \multicolumn{7}{|l|}{ Ultrasound } \\
\hline Cardiac & 2.5 & 3.6 & 3.44 & 1.9 & 2.9 & 0.0019 * \\
\hline Vascular & 3.1 & 2.8 & 3.1 & 3.0 & 3.0 & 0.923 \\
\hline Abdomen & 14.2 & 5.6 & 8.2 & 8.5 & 9.1 & $<0.0001$ * \\
\hline Any CT & 21.8 & 23.2 & 24.3 & 25.3 & 23.6 & 0.041 \\
\hline Brain & 17.0 & 18.0 & 21.3 & 22.9 & 19.7 & $<0.0001$ * \\
\hline Abdomen & 4.2 & 2.6 & 1.9 & 1.45 & 2.6 & $<0.0001$ * \\
\hline Thorax & 1.3 & 4.7 & 2.1 & 1.45 & 2.4 & $<0.0001$ * \\
\hline MRI brain & 3.0 & 2.0 & 2.6 & 1.9 & 2.4 & 0.051 \\
\hline Pulmonary scan & 2.5 & 2.7 & 2.4 & 2.2 & 2.5 & 0.68 \\
\hline Antibiotic-parenteral & 10.3 & 19.0 & 12.0 & 13.0 & 13.5 & $<0.0001$ * \\
\hline
\end{tabular}

OGD, oesphagogastroduodenoscopy; ERCP, endoscopic retrograde cholangiopancreatography. MRI, magnetic resonance imaging; CT, computed tomography. ${ }^{*}$ Bonferroni corrections applied-comparisons with $p<0.0038$ are considered significant.

significantly higher for GI (11.1\%), compared with respiratory $(3.5 \%)$, specialty $(4.4 \%)$, or GIM $(3.6 \%)$ consultants $(p<0.0001)$. Similarly the use of endoscopic retrograde cholangiopancreatography (ERCP) was significantly higher for GI $(7.4 \%)$ compared with respiratory $(0.1 \%)$, specialty $(0.6 \%)$, or GIM $(0.1 \%)$ consultants $(p<0.0001)$. Use of ultrasound showed some between consultant differences. Investigations, using cardiac ultrasound, were significantly higher for respiratory $(3.6 \%)$ and specialty groups $(3.4 \%)$ compared with GI $(2.5 \%)$ and GIM $(1.9 \%)(p=0.0019)$. Investigations, using abdominal ultrasound had an overall request rate of $9.1 \%$; these were used significantly more by GI $(14.2 \%)$ than respiratory consultants $(5.6 \%)(\mathrm{p}<0.0001)$.

The four consultant groupings differed significantly $(\mathrm{p}<0.0001)$ in their use of radiological resources. GIM and specialty used computed tomography (CT) of the brain comparatively more frequently, whereas CT of abdomen or thorax were singularly used, to a significantly greater extent than the remaining three groupings $(\mathrm{p}<0.0001)$, by GI and respiratory consultants respectively.

\section{Differences between consultant groups (HIPE discharge codes) (table 3) \\ Cardiovascular disease codes}

The frequency with which hypertension $(p=0.0017)$, congestive heart failure $(p=0.001)$, any heart failure/left ventricular failure $(\mathrm{LVF})(\mathrm{p}=0.0004)$, or atrial fibrillation $(\mathrm{p}=0.0001)$ were coded, were significantly different between the four consultant groupings (table 3 ). Hypertension was coded more frequently for patients admitted under specialty consultants. Heart failure was coded more frequently for patients cared for by GIM and respiratory consultants. Atrial fibrillation was coded more frequently by respiratory $(9.4 \%)$ compared with GI $(6.0 \%)$ consultants.

\section{Respiratory disease codes}

Pneumonia, a discharge code in $3.8 \%$ of patients, had similar discharge coding rates for all four groups. For any pneumonia diagnoses, and for any respiratory diagnosis, there were significant differences $(\mathrm{p}<0.0001)$ between consultant groups-being highest for respiratory $(6.7 \%$ and $24.3 \%$ respectively) and lowest for GI (3.6\% and $14.7 \%)$ respectively.

\section{Gastrointestinal disease codes}

Gastrointestinal codes included epigastic pain, gastritis, gastrointestinal haemorrhage, haematemesis, and peptic ulcer; considered together (5.4\% of admissions) there were differences $(p<0.001)$ for any GI coding between the groups. A GI code was more likely for GI consultants (7.5\%), compared with respiratory $(4.9 \%)$, specialty $(4.2 \%)$, and GIM (5.2\%) respectively. The alcohol related codes were alcohol withdrawal, alcohol abuse, alcoholic fatty liver,

Table 3 Diagnostic discharge codes by consultant group

\begin{tabular}{|c|c|c|c|c|c|c|}
\hline Discharge codes & GI & Respiratory & Specialty & GIM & Totalt & p Value \\
\hline Any coronary code & 7.3 & 9.7 & $9.2 \%$ & 9.1 & 8.8 & 0.026 \\
\hline Chest pain & 1.8 & 1.3 & 1.7 & 2.6 & 1.8 & 0.012 \\
\hline Hypertension & 11.5 & 13.2 & 15.2 & 12.8 & 13.2 & 0.0017 * \\
\hline Heart failure & 5.0 & 7.5 & 6.5 & 7.6 & 6.6 & $0.001 *$ \\
\hline Any CHF/LVF & 5.9 & 8.6 & 7.3 & 9.0 & 7.7 & 0.0004 * \\
\hline Any stroke code & 5.0 & 5.5 & 5.9 & 6.1 & 5.6 & 0.41 \\
\hline Atrial fibrillation & 6.0 & 9.4 & 8.3 & 8.9 & 8.1 & 0.0001 * \\
\hline UTI & 4.1 & 4.7 & 4.1 & 4.0 & 4.2 & 0.65 \\
\hline Pneumonia & 3.0 & 4.2 & 4.0 & 4.2 & 3.8 & 0.098 \\
\hline Any pneumonia & 3.6 & 6.7 & 5.2 & 5.0 & 5.1 & $<0.0001$ * \\
\hline Any respiratory disease & 14.7 & 24.3 & 19.3 & 21.6 & 19.9 & $<0.0001$ * \\
\hline Any gastrointestinal & 7.5 & 4.9 & 4.2 & 5.2 & 5.4 & $<0.0001$ * \\
\hline Any psychiatry code & 5.2 & 6.4 & 6.6 & 6.7 & 6.2 & 0.133 \\
\hline Any alcohol code & 7.8 & 5.2 & 4.9 & 6.1 & 6.0 & $<0.0001$ * \\
\hline Seizure code & 3.2 & 4.1 & 4.0 & 4.4 & 3.9 & 0.213 \\
\hline Any syncope code & 3.6 & 3.7 & 4.4 & 4.5 & 4.0 & 0.265 \\
\hline Any neoplasm & 6.3 & 11.3 & 4.7 & 4.5 & 6.7 & $<0.0001$ * \\
\hline Any anaemia & 3.5 & 2.4 & 3.5 & 3.2 & 3.2 & 0.093 \\
\hline Any diabetes & 5.7 & 5.7 & 10.0 & 5.6 & 6.8 & $<0.0001$ * \\
\hline
\end{tabular}

* Bonferroni corrections applied, comparisons with $\mathrm{p}<0.0026$ are considered significant. †Total does not add up to $100 \%$ as some patients can have multiple discharge codes. CHF, congestive heart failure; LVF, left ventricular failure; UTI, urinary tract infection. 
alcohol liver damage, alcohol cirrhosis of liver, and alcoholic hepatitis; considered together any alcohol related code $(6.0 \%$ of admissions) differed $(\mathrm{p}<0.0001)$ between the groups. Any alcohol code was more likely for GI consultants $(7.8 \%)$, compared with respiratory $(5.2 \%)$, specialty $(4.9 \%)$, and GIM $(6.1 \%)$ respectively.

\section{Other disease codes}

Codes of patients presenting with "funny turns" included syncope, dizziness and giddiness; these were present in $4.0 \%$ of admissions and rates of discharge coding did not differ between the consultant groups. Similarly, patients with seizure codes, present in $3.9 \%$ of cases, were similar between groups. Anaemia related codes included iron deficiency anaemia non-specific, and anaemia non-specific present in $3.2 \%$ of admissions did not differ between groups. Diabetes related codes included diabetes mellitus (DM) type l uncomplicated and DM type 2 uncomplicated; these were present in $6.8 \%$ of admissions. There were highly significant differences $(p<0.0001)$ in discharge coding of the latter related to consultant groups with higher rate for specialty $(10.0 \%)$, compared with the other groups $(5.7 \%)$. The overall reporting rate of any neoplasm at discharge was $6.7 \%$; there were highly significant $(\mathrm{p}<0.0001)$ differences between discharge coding rates of GI $(6.3 \%)$, specialty $(4.7 \%)$, respiratory $(11.3 \%)$, and GIM $(4.5 \%)$ respectively.

\section{DISCUSSION}

In this study comparing differences between hospital consultants, all trained in acute general medicine, the median LOS for acute general medical admissions was shorter when the patient was admitted under GIM consultants, compared with other consultant groups. Both GIM and respiratory consultants were less likely to have long stay patients $(>30$ days). After discharge from hospital, consultant groups differed in re-admission rates, being higher for respiratory consultants. Overall, patients who were re-admitted under the same consultant had a longer LOS than those who were re-admitted under a different consultant. However, patients re-admitted to hospital had the shortest LOS under GIM consultants. Endoscopy and GI radiological investigations were used predominately by GI consultants, while CT thorax and echo procedures were used most by respiratory consultants. Overall, the most used CT procedure was CT brain, being used most by GIM and specialty consultants. Respiratory diagnoses, malignancy, and atrial fibrillation were diagnosed more frequently by respiratory consultants; GI diagnoses more by GI consultants; diabetes and hypertension more by specialty consultants; and heart failure more by GIM and respiratory consultants.

We linked the hospital PAS and HIPE dataset to define a clinically useful database relating to emergency admissions to the division of medicine. Analysis of such data requires information that is comprehensive, accurate, and timely. Given the costs associated with such data collection, there is considerable literature using these type of data, and supporting their use for research, planning, and monitoring purposes. ${ }^{11}{ }^{12}$ However, the fact that the coding is done with a version of ICD-9-CM up to five years old means that advances in medical technology cannot always be captured in sufficient clinical detail. Moreover, complex clinical documentation, inexperienced coding personnel, and illegible handwritten medical record entries all contribute to inaccurate classification. $^{13-15}$ The Department of Health uses HIPE data to measure and compare hospitals' performance. The case mix directly influences funding given to a hospital, with more efficient hospitals being rewarded at the expense of the less efficient. Therefore, improvement in the quality of clinical coding is a desired goal to make such comparisons more meaningful and to provide a firm basis for both clinical and management decisions. In our study we found that the HIPE database was very powerful in predicting differences between hospital consultants in LOS, re-admission rates, use of resources, and disease coding.

We used a validated method in adjusting for the differences in physicians' LOS and readmission rate, with the frequency of comorbid diseases. ${ }^{10}$ Others have compared differences between physicians' severity adjusted LOS, and how through counselling and monitoring individual physicians' LOS may be reduced. ${ }^{16}$ Moreover, severity adjusted LOS provides a more accurate measure of LOS than unadjusted $\mathrm{LOS}_{,}{ }^{17}$ and the presence of comorbidity is significantly associated with longer LOS and hospital costs. ${ }^{18-20}$

We found great variation in LOS, re-admission rates, resource use, and diagnostic coding among physician groups, but comparatively little information to relate these variations systematically to specific physician characteristics. Others have reported greater test use by physicians trained in more academically oriented medical schools compared with those trained in less academically oriented settings. ${ }^{21}$ Differences between consultants' practice can be expected as, while defined standards for the management of several diseases exist, there is often a lack of consensus or guideline approaching the investigation of a patient with a given constellation of symptoms and signs. For those instances in which guidelines do exist, a decision to investigate beyond these may be taken either in response to additional findings, increasing demand for care, fear of litigation, ease of investigation availability, and the urge to make use of new technology. ${ }^{22-24}$ While it has been suggested that physicians overuse investigations in clinical practice without an associated increase in health status, ${ }^{22}$ it is generally accepted that before performing any test, the cost-benefit ratio is evaluated in each clinical situation, and if a diagnostic test is unlikely to change the patient's management, then the test should not be ordered. ${ }^{24} 25$ Standardised protocols and guidelines are therefore needed to formalise optimal practice in medical test ordering.

A study of emergency admissions under different consultant groups found that almost half of the acute in-patient bed days were inappropriate for acute care. ${ }^{26}$ Importantly, if discharge decisions are only made at the consultants' ward round, then the frequency of these ward rounds can influence LOS. ${ }^{27}$ However, one of the main contributors towards prolonged LOS is discharge delay pending placement into residential care or the provision of community support services. ${ }^{28}$ Early and frequent input from the social work department shortened LOS in a group of frail elderly patients requiring social care provision. ${ }^{29}$ Organisational strategy therefore seems to be an important potential tool for tackling the resource crisis, and underlines the effectiveness of a well coordinated multidisciplinary team. After discharge from hospital, high re-admission rates are sometimes considered to be the price for shorter inpatient stays among different consultant groups. ${ }^{30}$ Moreover, from a financial standpoint, re-admissions entail returns for the most expensive types of health services, acute inpatient care. ${ }^{31} 32$ However, it is generally accepted that many re-admissions may not be preventable, representing fresh events in patients with chronic illnesses and frequent comorbidity. ${ }^{33}$

A study of acute hospital admissions for end stage liver disease found that patients admitted under the care of a consultant gastroenterologist had a significantly shorter LOS (four compared with five days) and had less mortality, compared with patients admitted under the care of a nongastroenterologist. ${ }^{34}$ This difference persisted after adjustment for patients' age, morbidity, and number of procedures performed. Furthermore, costs of care were not significantly 
different between physician groups. ${ }^{34}$ A further study of 124 patients admitted acutely to hospital with upper gastrointestinal haemorrhage found that the median LOS for patients admitted under the GI service was significantly shorter (two compared with four days), and hospitalisation costs lower, than for patients admitted under the care of other physicians. ${ }^{35}$

It has been shown that patients hospitalised with acute heart failure under the care of cardiologists are more likely to undergo invasive procedures such as right heart catheterisation or cardiac catheterisation, than patients admitted under the care of general physicians. ${ }^{7}$ However, at 30 days there was no difference in adjusted survival between the groups. ${ }^{7}$ Similarly, others found that patients admitted with acute heart failure under the care of a cardiologist, had longer LOS and more invasive procedures, but were less likely to be readmitted with heart failure within six months compared with those admitted under the care of a general physician. ${ }^{36}$ Therefore, the implications for costs and survival differences among patients with congestive heart failure managed by cardiologists or general physicians require further study to understand how to best optimise care for these patients.

A study of 866 adults with severe COPD, including patients ventilated in an intensive care unit setting, found no difference in resource utilisation, hospital costs, or mortality whether treated by respiratory physicians or GIM physicians. ${ }^{37}$ Similar results were found for ambulatory patients with COPD. $^{38}$ A possible explanation for the lack of association between physician specialty and resource use or survival is that patients may have received similar treatment as a result of uniform practice protocols or guidelines. Moreover, as the choice of treatments for COPD is limited, differences in the type of care may not have led to differences in survival or hospital costs. Similarly, in a study of 260 patients admitted to hospital with diabetic ketoacidosis (DKA), after adjusting for case mix and severity of DKA, there was no difference in the LOS or diabetes related complications in those patients treated by endocrinologists compared with those treated by general physicians. ${ }^{39}$ However, the endocrinologist treated group had a lower readmission rate for DKA than the general physician treated group. ${ }^{39}$

There is growing evidence that, compared with specialists, hospitalist grade physicians can shorten the LOS and decrease inpatient costs while maintaining the quality of patient care. ${ }^{40}{ }^{41}$ Because the hospitalist model may offer a partial solution to the inflationary rise in hospital costs, it is being routinely considered as a model for teaching hospitals, and has prompted comparisons of outcomes and costs of generalist compared with specialist care. However, it should be emphasised that potential negative effects of the hospitalist model include depletion of the specialist workforce, de-skilling of other physicians, and physician burnout, making it unlikely to be an attractive long term career option.

In our study we found that the HIPE database was very powerful in predicting differences between hospital consultants in LOS, re-admission rates, resource use, and disease coding. We found little information to relate these differences to specific physician groups. Variations between consultants' practice may reflect a genuine special interest bias, or absence of definitive guidelines for the management of common acute medical conditions. It would be of interest to examine the extent to which protocols and guidelines could reduce such variations.

\footnotetext{
Authors' affiliations

E D Moloney, D Smith, D O'Riordan, B Silke, Division of Internal Medicine St James' Hospital, Dublin, Ireland
}

K Bennett, Department of Therapeutics and Pharmacology, Trinity College Dublin, Trinity Centre at St James' Hospital

Funding: none.

Conflicts of interest: none declared.

\section{REFERENCES}

1 Bernard AM, Shapiro LR, McMahon LF. The influence of attending physician subspecialization on hospital length of stay. Med Care 1990;28:170-4.

2 Hayward RA, Manning WG, McMahon LF, et al. Do attending or resident physician practice styles account for variations in hospital resource use. Med Care 1994;32:788-94.

3 Burns LR, Wholey DR. The effects of patient, hospital, and physician characteristics on length of stay and mortality. Med Care 1991;29:251-71.

4 Eisenberg J. Physician utilization: the state of research about physicians' practice patterns. Med Care 1985;23:461-83.

5 Greenfield S, Nelson EC, Zubkoff $M$, et al. Variations in resource utilization among medical specialties and systems of care. JAMA 1992;267:1624-30

6 Feinglass J, Martin GJ, Sen A. The financial effect of physician practice style on hospital resource use. Health Serv Res 1991;26:183-205.

7 Auerbach AD, Hamel MB, Davis RB, et al. Resource use and survival of patients hospitalised with congestive heart failure: differences in care by specialty of the attending physician. Ann Intern Med 2000;132:191-200.

8 Moloney ED, Silke B, Bennett K. Length of hospital stay, diagnoses and pattern of investigation following emergency admission to an Irish teaching hospital. Ir Med J 2004;97:170-2.

9 ESRI. Activity in acute public hospitals in Ireland 1990-1999. Dublin: ESRI, 2002.

10 Charlson ME, Pompei P, Ales KL, et al. A new method of classifying prognostic comorbidity in longitudinal studies: development and validation. J Chronic Dis 1987;40:373-83.

11 Bain MR, Chambers JW, Brewster DH. Routinely collected data in national and regional databases - an underused resource. J Public Health Med 1997:19:413-18.

12 Berkelman RL, Buehler JW. Public health surveillance of non-infectious chronic diseases: the potential to detect rapid changes in disease burden. Int J Epidemiol 1990; 19:628-35.

13 Maclntyre CR, Ackland MJ, Chandraraj EJ, et al. Accuracy of ICD-9-CM codes in hospital morbidity data, Victoria: implications for public health research. Aust N Z J Public Health 1997;21:477-82.

$14 \mathrm{O}^{\prime}$ Neill B. The hospital in-patient enquiry system - a study of data accuracy. Ir Med J 1982;75:238-9.

15 Smith MW. Hospital discharge diagnoses: how accurate are they and their international classification of disease (ICD) codes? N Z Med J 1989; 102:507-8.

16 Gross PA, Yost-Cataruozolo PE, DeMauro P, et al. Use of severity adjusted length of stay to modify physician practice patterns. Clin Perform Qual Health Care 1993; 1:23-8.

17 Gross PA, Cataruozolo P, DeMauro P, et al. Severity adjustment for length of stay: is it always necessary? Clin Perform Qual Health Care 1997;5:169-72.

18 Matsui K, Goldman L, Johnson PA, et al. Comorbidity as a correlate of length of stay for hospitalised patients with acute chest pain. J Gen Intern Med $1996 ; 11: 262-8$.

19 Ishizaki T, Imanaka Y, Oh E, et al. Association of hospital resource use with comorbidity status and patient age among hip fracture patients in Japan. Health Policy 2004;69:179-87.

20 Kinnunen T, Saynajakangas O, Tuuponen T, et al. Impact of comorbidities on the duration of COPD patients' hospital episodes. Respir Med 2003;97:143-6.

21 Epstein AM, Begg CB, McNeil BJ. The effects of physicians' training and personality on test ordering for ambulatory patients. Am J Public Health 1984;74:1271-3.

22 Winkens R, Dinant GJ. Rational cost effective use of investigations in clinical practice. BMJ 2002;324:783-5.

23 Epstein AM, McNeil BJ. Physician characteristics and organisational factors influencing use of ambulatory tests. Med Decis Making 1985;5:401-15.

24 Marik PE, Rakusin A, Sandhu SS. The impact of the accessibility of cranial CT scans on patient evaluation and management decisions. J Intern Med 1997;241:237-43.

25 Relman AS. Assessment and accountability: the third revolution in medical care. N Engl J Med 1988;318:1220-2.

26 Armstrong SH, Peden NR, Nimmo S, et al. Appropriateness of bed usage for inpatients admitted as emergencies to internal medicine services. Health Bull 2001;59:388-95.

27 Griffiths $M$, Walter WE, Acheson, eds. Variation in hospital stay after inguinal herniorrhaphy. BMJ 1979;i:787-9.

28 Black D, Pearson M. Average length of stay, delayed discharge and hospital congestion. BMJ 2002;325:610-11.

29 Fillit H, Howe JL, Fulop G, et al. Studies of hospital stays in the frail elderly and their relationship to the intensity of social work intervention. Soc Work Health Care 1992;18:1-22.

30 Jones J. Re-admission rates: the price of early discharge. Health Serv J 1986;96:825.

31 Anderson G, Steinberg E. Hospital re-admissions in the medicare population. N Engl J Med 1984;311:1349-53. 
32 Walter $\mathbf{R}$. The revolving door of hospital re-admissions. Caring 1998;17:56-9.

33 Moloney ED, Bennett K, Silke B. Patient and disease profile of emergency medical re-admissions to an Irish teaching hospital. Postgrad Med J 2004:80:470-4.

34 Ko CW, Kelley K, Meyer KE. Physician specialty and the outcomes and cost of admissions for end-stage liver disease. Am J Gastroenterol 2001;96:341 1-18.

35 Quirk DM, Barry MJ, Aserkoff B, et al. Physician specialty and variations in the cost of treating patients with acute upper gastrointestinal bleeding. Gastroenterology 1997:113:1443-8.

36 Reis SE, Holubkov R, Edmundowicz D, et al. Treatment of patients admitted to the hospital with congestive heart failure: specialty-related disparities in practice patterns and outcomes. J Am Coll Cardiol 1997;30:733-8.
37 Regueiro CR, Hamel MB, Davis RB, et al. A comparison of generalist and pulmonologist care for patients hospitalized with severe chronic obstructive pulmonary disease: resource intensity, hospital costs, and survival. Am J Med 1998; 105:366-72.

38 Strauss MJ, Conrad D, LoGerfo JP, et al. Cost and outcome of care for patients with chronic obstructive lung disease: analysis by physician specialty. Med Care 1986;24:915-24.

39 Levantan CS, Parasso MD, Jablonski KA, et al. Effect of physician specialty on outcomes in diabetic ketoacidosis. Diabetes Care 1999;22:1790-5.

40 Diamond HS, Goldberg E, Janosky JE. The effect of full-time faculty hospitalists on the efficiency of care at a community teaching hospital. Ann Intern Med 1998; 129:197-203

41 Wachter R, Katz P, Showstack J, et al. Re-organising an academic medical service. JAMA 1998;279:1560-5.

\section{IMAGES IN MEDICINE.}

\section{Starry sky appearance}

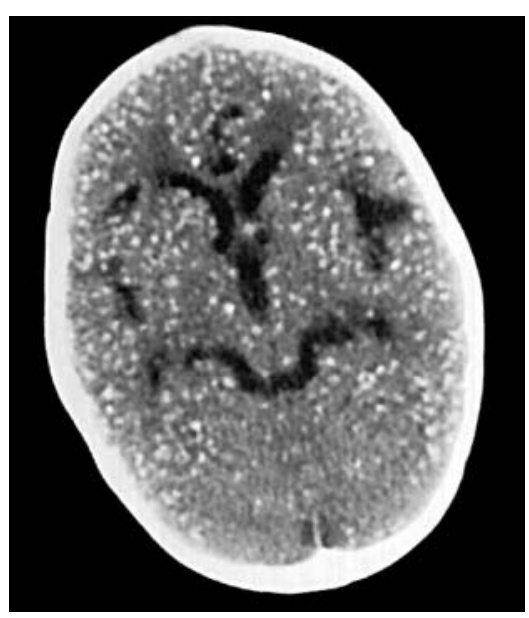

Figure 1 Computed tomography shows multiple intracranial calcifications, in both the cerebral and cerebellar hemispheres.
18 year old woman presented with a two month history of headache and two episodes of seizures in the past. On examination, she was afebrile, had no focal signs and no neurological deficit.

Computed tomography showed multiple intracranial hyperdense focii (calcifications), seen diffusedly distributed in both the cerebral and cerebellar hemispheres (fig 1). No evidence of any perilesional oedema or midline shift was seen. These widespread intra-parenchymal calcifications, giving a starry sky appearance on computed tomography are consistent with a diagnosis of neurocysticerosis.

Cysticercosis is the most common parasitic disease involving the brain. It is seen in both immuno-suppressed and immuno-competent people from endemic regions. It is caused by ingesting the ova of the pork tape worm (Taenia solium), through unwashed faecally contaminated vegetables or water. Humans are the inter-mediate hosts of Taenia solium. The location of involvement in brain can be parenchymal, intra-ventricular, or meningobasal. The diagnosis of cysticercosis is based on clinical, radiological, and serological indicators. Treatment is mostly medical (praziquantel), however surgical treatment may be required if ventricular obstruction is present.

K S Sodhi, N Khandelwal, S Suri Department of Radiodiagnosis and Imaging, Postgraduate Institute Of Medical Education And Research, Chandigarh, India

Correspondence to: Dr K S Sodhi, Department of Radiodiagnosis and Imaging, PGIMER, Chandigarh 160012, India; sodhiks@rediffmail.com 